\title{
Path-Dependent Option Valuation When the Underlying Path Is Discontinuous
}

\author{
Chunsheng Zhou ${ }^{\dagger}$
}

March 1997

${ }^{\dagger}$ Federal Reserve Board, Mail Stop 91, Washington, DC 20551. Tel: (202) 452-3328, Fax: (202) 452-5296, E-mail: czhou@frb.gov. The author thanks Katherine Allen for research assistance. The analysis and conclusions set forth in this paper are those of the author and do not indicate concurrence by other members of the research staff, by the Board of Governors, or by the Federal Reserve Banks. 


\begin{abstract}
The payoffs of path-dependent options depend not only on the final values, but also on the sample paths of the prices of the underlying assets. A rigorous modeling of the underlying asset price processes which can appropriately describe the sample paths is therefore critical for pricing path-dependent options.

This paper allows for discontinuities in the sample paths of the underlying asset prices by assuming that these prices follow jump diffusion processes. A general yet tractable approach is presented to value a variety of path-dependent options with discontinuous processes. The numerical examples show that ignoring the jump risk may lead to serious biases in pathdependent option pricing.
\end{abstract}


Jumps are an important feature of the price processes of financial assets, and are especially pronounced for certain types of assets, such as small-cap stocks and exchange rates. ${ }^{1}$ For this reason, Merton's (1976) groundbreaking work, which explicitly admits jumps in the underlying asset prices for pricing standard European options, has generated a profound impact on the finance profession.

Merton's work was done a couple of decades ago. In recent years, the variety of new option contracts has increased enormously. Many types of so-called exotic options are now popular items in the over-the-counter market. Most exotic options, such as barrier options, lookbacks, Asian options, American capped options, and many others, are path-dependent, that is, their payoffs depend not only on the final values, but also on the sample paths of the underlying asset prices. For these options, a rigorous consideration of jumps in the sample paths of the underlying asset prices seems more important than for the standard non-pathdependent options in Merton's world. But these new path-dependent options are often so complicated that they cannot be priced in Merton's framework. For this reason, this paper presents a new general yet tractable approach to value a variety of path-dependent options whose underlying prices follow jump-diffusion processes. ${ }^{2}$

If a jump-diffusion process of the underlying asset price is misspecified as a pure diffusion process, what will happen to the pricing performance of the option pricing model? For a given option, say a down-and-out call, will it be overpriced, or underpriced by the model due to the misspecification of the price process? To answer these questions, several numerical examples are provided on barrier options and lookback options. The examples show that for the path-dependent options, ignoring the jump risk often leads to serious biases in the pricing of both short maturity and long maturity options. The examples also show some interesting and sometimes surprising maturity patterns of pricing errors

\footnotetext{
${ }^{1}$ See, Kon (1984), Ball and Torous (1985), Jorion (1988), and Bates (1991, 1996), Das, Foresi and Sundaram(1996).

${ }^{2}$ In a recent paper, Amin (1993) provided a discrete time approach to value one kind of path dependent option - an American option under jump diffusion processes. The approach presented here is much different from Amin's approach.
} 
caused by the misspecification. For instance, misspecifying a jump-diffusion process as a diffusion process will understate the knock-out probability for short-maturity options, but significantly overstate the knock-out probability for long-maturity options. As a result, this misspecification often overvalues short maturity down-and-out calls, but underprices most long maturity down-and-out calls.

The rest of this paper is organized as follows: The next section presents a general approach to valuing path-dependent options with jump-diffusion processes. Sections 2 and 3 provide numerical examples to show the impact of the jump risk on the prices of barrier options and lookback options. Section 4 concludes.

\section{The Model}

This section presents a general jump-diffusion model to value various path-dependent options. The model is based on the following assumptions.

Assumption 1: The dynamics of the underlying asset price $S$ are governed by the jump-diffusion process

$$
d S / S=(\mu-\lambda \nu) d t+\sigma d Z+(\Pi-1) d Y
$$

where

$\mu, \nu, \lambda$, and $\sigma$ are positive constants;

$Z$ is a standard Brownian motion;

$d Y$ is a Poisson process with intensity parameter $\lambda$;

$\Pi>0$ is the jump amplitude with expected value equal to $\nu+1$, and

$d Z, d Y$, and $\Pi$ are mutually independent.

Because $\nu$ equals the expected value of the jump component $(\Pi-1), \mu$ in the above equation represents the expected instantaneous rate of change of the asset price $S$.

We assume that $\Pi$ is an i.i.d. log-normal random variable, such that

$$
\ln (\Pi) \sim N\left(\mu_{\pi}, \sigma_{\pi}^{2}\right)
$$


This assumption implies that

$$
\nu:=E[\Pi-1]=\exp \left(\mu_{\pi}+\sigma_{\pi}^{2} / 2\right)-1
$$

The diffusion process in equation (1) characterizes the "normal" vibration in the asset price, due to gradual changes in economic conditions or the arrival of new information which causes marginal changes in the asset value. The jump component describes the "abnormal" variations in the asset price due to the arrival of important new information.

Assumption 2: The capital asset pricing model (CAPM) holds for equilibrium security returns and the jump component of the underlying asset price $S$ in equation (1) is purely asset-specific and is uncorrelated with the market.

This paper considers the options of individual stocks. According to Merton (1976), there generally does not exist a set of portfolio weights that will eliminate the "jump" risk. A Black-Scholes hedge will not be riskless even in a continuous-time setup. To validate the Black-Scholes "risk-neutral" argument, some extra restrictions on the economy and the jump process must be imposed. If the jump component represents nonsystematic risk, a portfolio which removes the risk of diffusion component (i.e., $d Z$ does not appear in the return process of the portfolio) will have a zero "beta." By the CAPM, the expected return on that portfolio must equal the riskless rate. The jump risk will therefore not receive a risk premium. Assumption 2 has been widely used as a first-order approximation for jumps in the prices of individual stocks, even though it is not a valid assumption for jumps in market indexes.

With an increase in complexity, Assumption 2 may be replaced by some alternative assumptions. For example, one can assume that jumps are systematic and that economic agents have some specific preferences so that risk premium for jumps can be determined in the model. Bates $(1991,1996)$ finds that under a traditional assumption of preferences, the 'risk-neutral' movement of the underlying asset price follows a jump-diffusion process similar to the one under Assumption 2, so the basic approach in this paper is still valid for handling nonsystematic jumps. In other words, Assumption 2 simplifies the model, but does not change the main conclusions of this paper. 
Denote $W\left(\Gamma ; S_{t}: 0 \leq t \leq T\right)$ as the final payoff of a path-dependent option contract, where $\Gamma$ is the set of relevant parameters and $\left(S_{t}: 0 \leq t \leq T\right)$ is the time-path of the price of the underlying asset. Using a standard risk-neutrality approach, we have

Lemma 1 The option price $F(\Gamma, T)$ is given by

$$
F=\exp (-r T) E^{Q}\left[W\left(\Gamma ; S_{t}: 0 \leq t \leq T\right)\right]
$$

where $E^{Q}$ represents the expectation under the equivalent martingale measure $Q$ conditioning on information currently available. Under this measure,

$$
d S / S=(r-\lambda \nu) d t+\sigma d Z+(\Pi-1) d Y
$$

Equation (4) can be rewritten as:

$$
d \ln (S)=\left(r-\sigma^{2} / 2-\lambda \nu\right) d t+\sigma d Z+\ln (\Pi) d Y .
$$

This lemma provides a general framework for option valuation. The next two sections will use this lemma to value barrier options and lookback options.

\section{Pricing Barrier Options}

Barrier options are path-dependent options which are either activated (knocked-in) or terminated (knocked-out) if the underlying asset value reaches or passes a specified trigger level, say $H$, between inception and expiration. ${ }^{3}$ After activation and before extinction, barrier options behave identically to standard European-style options. These options have become increasingly popular in recent years. Less expensive than standard options, they may provide the appropriate hedge in a number of situations. For example, a down-and-out call with a low barrier offers an inexpensive protection against a big rise in the underlying asset price.

\footnotetext{
${ }^{3}$ For detailed discussions of various barrier options, see, for example, Rubinstein and Reiner (1991), Heyne and Harry (1994), Boyle and Lau (1994), Ritchken (1995), and Rich (1994).
} 
For barrier options, jumps not only affect the distribution of the value of the underlying asset at the maturity of an option, but also influence the barrier crossing probability of the underlying asset. In this section, we investigate how jumps change barrier crossing probabilities and option values.

\subsection{Methodology}

We use the down-and-out call option as an example to illustrate the effect of the jump risk on barrier option pricing. The option ceases to exist when the underlying asset price $S$ hits or falls below a constant barrier $H$. We assume that the maturity of the option is $T$ and the exercise price of the option is $X$, where $X>H$. If the underlying asset price follows a standard log-normal diffusion process, the option price can be expressed analytically as

$$
D A O C(S, X, H, r, \sigma, T)=B S C(S, X, r, \sigma, T)-\left(\frac{H}{S}\right)^{\gamma-1} B S C\left(\frac{H^{2}}{S}, X, r, \sigma, T\right),
$$

where $D A O C$ is the down-and-out call price, $B S C$ is the Black-Scholes standard European call price, and $\gamma=2 r /\left(\sigma^{2}\right)$. Other notation is defined as before.

We now consider the prices of down-and-out call options with jump diffusion processes and compare them with the prices of the corresponding options with log-normal diffusion processes obtained through equation (6).

Denote $\tau$ as the first passage time for the underlying asset price $S$ crossing the barrier $H$, that is

$$
\tau:=\inf \left\{t \mid S_{t} \leq H, t \geq 0\right\}
$$

If $\tau \leq T$, the option is knocked-out before its maturity and becomes worthless. Otherwise, the option is not knocked out during its life and receives a payment at its maturity just like a standard European call. The final payoff to the option can be expressed as:

$$
W_{T}= \begin{cases}0, & \text { if } \tau \leq T \\ \max \left(0, S_{T}-X\right) & \text { otherwise }\end{cases}
$$

It follows immediately that

$$
D A O C(S, X, H ; T)=\exp (-r T) E^{Q}\left[W_{T}\right]
$$




$$
=\exp (-r T) E^{Q}\left[\max \left(0, S_{T}-X\right) \mid \tau>T\right] Q(\tau>T)
$$

where $Q(\tau>T)$ is the probability that the first passage time $\tau$ is bigger than the maturity of option $T$ under the equivalent martingale measure $Q$.

Theorem 1 Divide the time interval $[0, T]$ into $n$ equal subperiods and define

$$
\begin{gathered}
t_{i}=\frac{i}{n} T \\
\Omega=\left\{S_{t_{i}}^{*}>H, i=1,2, \cdots, n\right\},
\end{gathered}
$$

where $S_{t_{i}}^{*}$ is defined recursively as

$$
\begin{gathered}
S_{t_{0}}^{*}=S, \\
\ln \left(S_{t_{i}}^{*}\right)-\ln \left(S_{t_{i-1}}^{*}\right)=s_{i}+y_{i} \cdot \pi_{i}, \quad i=1,2, \cdots, n .
\end{gathered}
$$

Here $s_{i}, y_{i}$, and $\pi_{i}$ are mutually and serially independent random variables drawn from

$$
\begin{gathered}
s_{i} \sim N\left(\left(r-\sigma^{2} / 2-\lambda \nu\right) T / n, \sigma^{2} \cdot T / n\right), \\
\pi_{i} \sim N\left(\mu_{\pi}, \sigma_{\pi}^{2}\right),
\end{gathered}
$$

and

$$
y_{i}=\left\{\begin{array}{l}
0, \text { with prob. } 1-\lambda \cdot T / n \\
1, \text { with prob. } \lambda \cdot T / n
\end{array}\right.
$$

Then the option price $D A O C(S, X, H ; T)$ given in equation (8) can be expressed as

$$
D A O C(S, X, H ; T)=\exp (-r T) \lim _{n \rightarrow \infty} E^{Q}\left[\max \left(0, S_{T}^{*}-X\right) \mid \Omega\right] Q(\Omega)
$$

Briefly speaking, the theorem holds because in a very small time period, no more than one jump can occur almost surely and the diffusion process cannot move a long distance. The theorem can be easily extended to value options with time-varying barriers or multiple barriers. The proof of the theorem is outlined in the appendix.

Theorem 1 provides a straightforward framework to price barrier options. The following is a Monte Carlo valuation procedure based on this framework, which involves nothing more complex than making random draws from some simple distributions. 
- Step (1). Divide the time interval $[0, T]$ into $n$ equal subperiods for a sufficiently large ${ }^{4}$ $n$ and denote $t_{i}:=T \cdot i / n$.

- Step (2). Do Monte Carlo replications for $M(j=1,2, \cdots, M)$ times. For each $j$, run the following sub-procedures for $i=1, \cdots, n$.

a) Generate a series of mutually and serially independent random vectors $\left(s_{i}, \pi_{i}, y_{i}\right)$ as described in Theorem 1 . Let $S_{t_{0}}^{*}=X$ and calculate $\ln \left(S_{t_{i}}^{*}\right)$ or $S_{t_{i}}^{*}$ according to the formula

$$
\ln \left(S_{t_{i}}^{*}\right)=\ln \left(S_{t_{i-1}}^{*}\right)+s_{i}+y_{i} \cdot \pi_{i}
$$

If $S_{t_{i}}^{*} \leq H$, let $W_{j}=0$ and go to the next $j$.

b) Let $W_{j}=\max \left(0, S_{t_{n}}^{*}-X\right)$ and go to the next $j$.

- Step (3). Let $C=\exp (-r T) \sum_{j=1}^{M} W_{j} / M$. $C$ will be a numerical solution to the option price.

\subsection{Numerical Simulations}

The following numerical illustrations show some rich implications of the model. In these illustrations, the current stock price $S$ is chosen as $\$ 20.00$ and the barrier price $H$ is $\$ 16.00$. Equation (5) implies that the volatility of underlying asset price growth $d \ln (S)$ is

$$
\sigma_{S}^{2}:=\operatorname{Var}(d \ln (S)) / d t=\sigma^{2}+\lambda \cdot \sigma_{\pi}^{2},
$$

\footnotetext{
${ }^{4}$ The appropriate choice of $n$ depends on the remaining maturity of the option $T$ and the distance between the current underlying price and the barrier $|\ln (S)-\ln (H)|$. If $T$ and $|\ln (S)-\ln (H)|$ are small, then to avoid the problem that the barrier is 'hit but missed' and to increase the pricing accuracy (see, e.g., Geman and Yor 1996), the step size $T / n$ should also be very small. Interestingly, we find that simulated option prices are less sensitive to step sizes under jump processes than under diffusion processes. This is because when the step size is small enough, it is unlikely that there is more than one jump in one step. Following Geman and Yor (1996), we choose the step size as 1/4 day. It takes about one minute to value a one-month option with a SPARC 20 computer if we perform 10,000 Monte Carlo paths. To improve the efficiency of Monte Carlo simulations, some numerical techniques may be used. See, for example, Chidambaran and Figlewski (1995) for a detailed discussion.
} 
if $\mu_{\pi}=0$. For convenience, $\mu_{\pi}$ will be set to zero in our numerical simulations. The value of $\sigma_{S}^{2}$ will be kept as a constant so that the changes in option values are really from the changes in the relative importance of the jump component rather than from the changes in the overall volatility of the underlying asset price. We use the following data (expressed at a monthly frequency) in simulations:

$\sigma_{S}=0.10:$ Monthly volatility of asset prices is 10 percent. This implies approximately a 35 percent annual volatility.

$\lambda=0.03:$ On average, there are 0.03 jumps in a month or approximately one jump every three years. Jumps are rare events in our simulations.

$\sigma_{\pi}^{2}=0.10$. As argued by Merton, jumps are rare events which cause substantial changes in asset prices. Assuming $\sigma_{\pi}^{2}=0.10$ implies approximately a 30 percent standard deviation of the log change in the asset price caused by a jump. For rare (on average, once every three years in this case) and substantial jumps, this number does not seem implausible. Certain kind of growth stocks may have more volatile jumps. For comparison reason, $\sigma_{\pi}^{2}=0.25$ will also be used in simulations.

The main purpose of our numerical simulations is to provide readers with some flavor or conceptual insight about the potential impact of the jump risk on option prices. These simulations are not substitutes for further empirical studies. A rigorous empirical verification of the model is helpful, and is best treated as a subject for a separate paper.

\subsubsection{At-the-Money Call Options}

Table 1 illustrates the effect of the jump risk on the price of at-the-money call options with the volatility of the underlying asset price remaining constant. For down-and-out call options, jumps generally lower the price of short maturity options, while increasing the value of long maturity options. For example, the price of a $1 / 2$-month at-the-money downand-out call option with $\sigma_{\pi}^{2}=0.25$ is about $\$ 0.365$, but a similar option with the log-normal diffusion process is valued at as much as $\$ 0.589$. On the other hand, the price of a two-year at-the-money down-and-out call with $\sigma_{\pi}^{2}=0.25$ is about $\$ 4.19$, but a similar option with 
a log-normal diffusion process is only worth $\$ 3.79$. For standard at-the-money European calls, the jump risk consistently lowers the option prices over various maturities, but such effects become less important when option maturities are longer. The table suggests that the jump risk may be far more important than the second order effects in the valuation of path-dependent options. For barrier options, the effects of the jump risk option prices do not disappear with the lengthening of option maturities. Ignoring the jump risk may lead to large biases in option prices even if the volatilities of the underlying asset prices are correctly estimated.

The finding of Table 1 that the relation between option prices over short maturities is reversed over longer maturities is surprising. Since this result is not found for the prices of standard European options, it likely has something to do with knock-out probabilities. A detailed analysis is in order.

Table 2 shows the knock-out probabilities of options with different volatilities of the jump amplitude, $\sigma_{\pi}^{2}$. The table illustrates a pattern of knock-out probabilities which roughly matches the pattern of the prices of down-and-out call options: Holding constant the volatility of the underlying asset price $S$, over short maturities, an option with a more volatile jump component is more likely to be knocked-out than is an option with a more volatile diffusion component; over longer maturities, the relation is reversed: An increase in the jump volatility $\sigma_{\pi}$ can substantially reduce the knock-out probability of long-term options. Consider a two year knock-out call as an example. Under our parametric specification, when $\sigma_{\pi}=0$, the knock-out probability is 0.65 , but when $\sigma_{\pi}=0.25$, this probability is only 0.45 .

Because a diffusion component is much less likely to produce a large change in the asset price $S$ over a short period, a short-maturity option with a more volatile jump component is more likely to be knocked out than is a same maturity option with a less volatile jump component. What is interesting here is that for long maturity options, a high jump volatility is generally associated with a low knock-out probability. We now outline some intuition for this result. 
For a given $T>0$ which is not very small in magnitude, an increase in the volatility of a diffusion process can substantially increase the knock-out probability during $[0, T]$. However, for a jump process, the effect of the jump size volatility $\sigma_{\pi}$ on the knock-out probability is largely limited by the jump intensity $\lambda$. If $\lambda$ is very small such that $\lambda T$ is also small, the probability that there is at least one jump in period $[0, T]$ is approximately $\lambda T$. As a result, no matter how large the jump size volatility $\sigma_{\pi}^{2}$ is, the knock-out probability in period $[0, T]$ caused by the jump process is always smaller than $\lambda T$, even though $\lambda T$ is already small. In this case, an increase in $\sigma_{\pi}^{2}$ mainly affects the value of asset at knock-out time but has a very small effect on the knock-out probability. This intuition is made more rigorous in the following example.

Consider two extreme $S$ processes for illustration. The first one is a pure diffusion process with the volatility $\sigma^{2}$ and the second one is a pure jump process with a small jump intensity $\lambda$ and a large volatility of jump amplitude $\sigma_{\pi}^{2}:=\operatorname{Var}(\ln (\Pi))$. I assume that $\sigma^{2}=\lambda \cdot \sigma_{\pi}^{2}=0.01$, which is the same as the volatility of $\ln (S)$ used in Table 2 .

Denote $D(T)$ as the cumulative distribution function of the first passage time to the barrier $H$ for the pure diffusion process and denote $J(T)$ as the cumulative distribution function of the first passage time to the barrier for the pure jump process, where $T$ is the maturity time. Using the result of Harrison (1990), we have

$$
\begin{aligned}
D(T)= & N\left(-\frac{\ln (S / H)+\left(r-\sigma^{2} / 2\right) T}{\sigma \sqrt{T}}\right) \\
& +(S / H)^{\left(1-\frac{2 r}{\sigma^{2}}\right)} N\left(-\frac{\ln (S / H)-\left(r-\sigma^{2} / 2\right) T}{\sigma \sqrt{T}}\right) .
\end{aligned}
$$

Assuming that $S=20$ at time zero and $H=16$ as in Table 2, we obtain immediately that $D(1 / 4)=0.00001$ and $D(18)=0.599$.

There is no explicit expression for $J(T)$. However, in a pure jump process with a positive drift, crossing the barrier must be caused by jumps. Assume that $\lambda=0.03$ and that $\sigma_{\pi}^{2}=0.01 / 0.03=1 / 3$. If $T=1 / 4$ (one month), then the probability of one jump in $[0, T]$ is about $\lambda T=0.0075$ and the probability of two or more jumps in $[0, T]$ is small enough to ignore. If a jump occurs at time $t<1 / 4$ and there are no other jumps before $t$, 
the probability that $S_{t}$ falls to or below $H$ is

$$
N\left(-\ln (S / H) / \sigma_{\pi}\right)=N(-\ln (20 / 16) / \sqrt{1 / 3})=0.35
$$

As a result, we have $J(1 / 4) \approx 0.0075 \times 0.35=0.003$.

Now let's consider $T=18$. The probability that there is no jump in the time interval $[0, T]$ is

$$
\exp (-\lambda T)=\exp (-0.03 \times 18)=0.583
$$

That is, the probability that there are one or more jumps in $[0, T]$ is $1-0.583=0.417$. Denote $d<1$ as the conditional probability of crossing the barrier if there are jumps. Then we have

$$
J(18)=0.417 \cdot d \ll 0.599
$$

From the above examples, we see that $J(1 / 4) \gg D(1 / 4)$ and that $J(18) \ll D(18)$. That is, a jump process is more likely than is a diffusion process to cause a knock-out over a short horizon but less likely to cause a knock-out over a long horizon.

\subsubsection{In-the-Money and Out-of-the-Money Options}

Tables 3 and 4 report the example prices of the in-the-money and the out-of-the-money call options.

Let's discuss in-the-money options first. For options with very short maturities, knockout probabilities are very low so that the prices of the standard options and the corresponding barrier options are very close.

As maturities become longer, the prices of the standard options and the barrier options display different patterns. Similar to the at-the-money cases shown in Table 1, the values of the in-the-money standard European call options with jumps tend to be lower than those of the corresponding maturity options with continuous diffusion processes. Holding constant the volatility of the underlying asset price, the prices of options become insensitive to the jump risk when option maturities are sufficiently long. For the barrier options, jumps reduce the option values in the medium range of maturities, same as that which happens to 
the standard in-the-money call options. This is because with these maturities, the knockout probabilities have not become large enough to change the properties of barrier options inherited from their standard counterparts. As the maturities become long enough, the knock-out probabilities of options with diffusion processes become much larger than the knock-out probabilities of options with jumps, holding $\sigma_{S}^{2}$ constant (See Table 2). As a result, the options with more volatile jump components become more valuable.

We now consider out-of-the-money calls.

For both the standard and the down-and-out out-of-the-money calls, jumps generally raise the value of options near expiration. This is because the underlying asset prices with more volatile jump components are more likely to move a sufficient distance to pass the strike price $X(X>S)$ in a short period, as discussed earlier.

As maturities get longer, the standard out-of-the-money calls with jumps become less valuable, because as was discussed previously, the diffusion processes are more likely to pass a threshold level ( $X$ in this situation) in a relatively long period. For down-and-out calls, the situation is more complicated. Jumps have two opposite effects on out-of-themoney option values. On the one hand, similar to that which occurs with the standard out-of-the-money calls, jumps tend to lower option values by reducing the probability that the underlying asset will cross the strike price $X$. On the other hand, jumps may increase option values by reducing knock-out probabilities, as shown in Table 2. The net impact depends on the relative importance of these two effects. In the example shown in Table 4, the first effect dominates over medium horizons and the second effect is more important over long horizons. As a result, jumps reduce the prices of down-and-out out-of-the-money calls with medium maturities but increase the prices of options with long maturities. 


\section{Pricing Lookback Options}

Lookback options give the holder the right, at maturity, to exercise the option at the most favorable price reached by the underlying asset during the life of the option. ${ }^{5}$ Take a lookback call option as an example. If $S_{m i n}$ is the minimum price reached and $S_{T}$ is the asset price at the maturity of the option, $T$, the payoff from the option is $S_{T}-S_{m i n}$. According to Lemma 1 , the price of this option is $\exp (-r T) E^{Q}\left[S_{T}-S_{m i n}\right]$.

Theorem 2 Using the same approach as in Theorem 1, the price of a lookback option can be expressed as

$$
L B(S, S M ; T)=\exp (-r T) \lim _{n \rightarrow \infty} E^{Q}\left[S_{T}^{*}-\min \left(S M ; S_{t_{0}}^{*}, S_{t_{1}}^{*}, \cdots, S_{t_{n}}^{*}\right)\right]
$$

where $S M$ is the minimum asset price reached to date and all other notation is defined as in Theorem 1.

The proof of this theorem is similar to that of Theorem 1. Based on this theorem, a numerical approach similar to the one used to value barrier options can be used to value lookback options.

Table 5 presents a numerical example to illustrate the effects of jumps on lookbacks. In this table, the volatility of stock price $\sigma_{S}^{2}$ is fixed at a constant level 0.01. The Table shows that jumps generally reduce the price of lookback call options. For short maturity lookbacks, jumps have a relatively small impact on option prices, but when option maturities get relatively long, jumps will affect option prices significantly. More specifically, in the numerical example shown here, a 1/2-month lookback call with log-normal process is worth about $\$ 2.12$. With a jump-diffusion process, such an option is valued at $\$ 2.08$ if $\sigma_{\pi}^{2}=0.10$ and at $\$ 2.06 \sigma_{\pi}^{2}=0.25$. If the maturity of a lookback call becomes one year, its price is about $\$ 5.64$ with a log-normal process and becomes as low as $\$ 4.74$ when jumps are present $\left(\lambda=0.03\right.$ and $\left.\sigma_{\pi}^{2}=0.25\right)$.

\footnotetext{
${ }^{5}$ For detailed discussions of lookback options, see, for example, Conze and Viswanathan (1991) and Goldman, Sosin, and Gatto (1979).
} 


\section{Conclusions}

This paper presents a general framework for valuing a variety of path-dependent exotic options in the presence of jumps in the underlying price process. It shows that ignoring jumps may lead to seriously biased options prices. For instance, for at-the-money downand-out barrier calls, ignoring jumps will lead to upward biases in option prices with short maturities, but downward biases in option prices with long maturities. For lookback call options, ignoring jumps will often lead to upward biases in options prices, especially for those with relatively long maturities. The biases are generally significant in magnitude.

\section{Appendix}

This appendix outlines the proof of Theorem 1 .

Proof of Theorem 1: Equation (5) implies that

$$
\ln \left(S_{t_{i}}\right)-\ln \left(S_{t_{i-1}}\right)=s_{i}+\sum_{j=0}^{\kappa_{i}} \pi_{i j},
$$

where

$$
\begin{gathered}
s_{i} \sim N\left(\left(r-\sigma^{2} / 2-\lambda \nu\right) T / n, \sigma^{2} \cdot T / n\right), \\
\pi_{i j} \sim N\left(\mu_{\pi}, \sigma_{\pi}^{2}\right),
\end{gathered}
$$

and

$$
\kappa_{i}=k, \text { with prob. } \lambda^{k} \cdot \frac{\exp (-\lambda \cdot T / n)}{k !} \cdot(T / n)^{k}, k=0,1, \cdots .
$$

Since $\operatorname{Prob}\left(\kappa_{i}=k\right)=o(T / n)$ for $k>1$, by the definitions of $S_{t_{i}}^{*}$ and $\Omega$, we have

$$
\begin{aligned}
D A O C(S, X, H ; T)= & \exp (-r T) E^{Q}\left[\max \left(0, S_{T}-X\right) \mid \tau>T\right] Q(\tau>T) \\
= & \lim _{n \rightarrow \infty} \exp (-r T) E^{Q}\left[\max \left(0, S_{T}-X\right) \mid S_{t_{i}}>H, i=1, \cdots, n\right] \\
& \cdot Q\left(S_{t_{i}}>H, i=1, \cdots, n\right) \\
= & \lim _{n \rightarrow \infty} \exp (-r T) E^{Q}\left[\max \left(0, S_{T}^{*}-X\right) \mid S_{t_{i}}^{*}>H, i=1, \cdots, n\right] \\
& \cdot Q\left(S_{t_{i}}^{*}>H, i=1, \cdots, n\right) \\
= & \lim _{n \rightarrow \infty} \exp (-r T) E^{Q}\left[\max \left(0, S_{T}^{*}-X\right) \mid \Omega\right] Q(\Omega) .
\end{aligned}
$$




\section{References}

[1] Amin, K.I.(1993): "Jump diffusion option valuation in discrete time," Journal of Finance 48, 1833-1863.

[2] Bates, D.S.(1991): "The crash of '87: Was it expected? The evidence from options markets," Journal of Finance 46, 1009-1044.

[3] Bates, D.S.(1996): "Jumps and stochastic volatility: Exchange rate processes implicit in Deutsche Mark options," Review of Financial Studies 9, 69-107.

[4] Ball, C. and W. Torous(1985): "On jumps in common stock prices and their impact on call option pricing," Journal of Finance 40, 155-173.

[5] Black, F.(1975): "Fact and fantasy in the use of options," Financial Analysts Journal 31 (July/August 1975), 36-72.

[6] Boyle, P.P. and S.H. Lau(1994): "Bumping up against the barrier with the binomial method," Journal of Derivatives, Summer 1994, 6-14.

[7] Chidambaran, N.K. and S. Figlewski(1995): "Streamlining Monte Carlo simulation with the quasi-analytic method: Analysis of a path-dependent option strategy," Journal of Derivatives, Winter 1995, 29-52.

[8] Conze, A. and Viswanathan(1991): "Path dependent options: The case of lookback options," Journal of Finance 46, 1893-1907.

[9] Cox, J.C. and S.A. Ross(1976): "The valuation of options for alternative stochastic processes," Journal of Financial Economics 3, 145-166.

[10] Das, S.R., S. Foresi, and R.K. Sundaram(1996): "Modeling asset price processes in finance," preprint.

[11] Geman, H. and M. Yor(1996): "Pricing and hedging double-barrier options: A probabilistic approach," Mathematical Finance 6, 365-378. 
[12] Goldman, M.B., H.B. Sosin, and M.A. Gatto(1979): "Path dependent options: 'Buy at the low, sell at the high'," Journal of Finance 34, 1111-1127.

[13] Harrison, J.M.(1990): Brownian motion and stochastic flow systems, Krieger Publishing Company: Florida.

[14] Heynen, H. and H. Kat(1994): Crossing barriers, Risk, June 1994, 46-51.

[15] Jarrow, R.A. and A. Rudd(1983): Option Pricing, Irwin.

[16] Jorion, P.(1988): "On jump processes in the foreign exchange and stock markets," Review of Financial Studies 1, 427-445.

[17] Kon, S.J.(1984): "Models of stock returns-A comparison," Journal of Finance 39, 147-165.

[18] Merton, R.C.(1976a): "Option pricing when underlying stock returns are discontinuous," Journal of Financial Economics 3, 125-144.

[19] Merton, R.C.(1976b): "The impact on option pricing of specification error in the underlying stock price returns," Journal of Finance 31, 333-350.

[20] Rich, D.R.(1994): "The mathematical foundations of barrier option-pricing theory," Advances in Futures and Options Research 7, 267-312.

[21] Ritchken, P.(1995): “On pricing barrier options," Journal of Derivatives, Winter 1995, 19-28.

[22] Rubinstein, M. and E. Reiner(1991): Breaking down the barriers, Risk, September 1991, 28-35. 
Table 1: Prices of At-the-Money Call Options (Monthly Frenquency). $S=20$, $X=20, \lambda=0.03, \mu_{\pi}=0, \sigma_{S}=0.1, \sigma^{2}=\sigma_{S}^{2}-\lambda \sigma_{\pi}^{2}$.

\begin{tabular}{cccccccccccc}
\hline \hline Maturity & $1 / 4$ & $1 / 2$ & 1 & 2 & 3 & 6 & 9 & 12 & 18 & 24 \\
\hline \multicolumn{8}{c}{ Down-and-Out Call Options $(H=16)$} \\
$\sigma_{\pi}^{2}=0.00$ & 0.411 & 0.589 & 0.846 & 1.223 & 1.518 & 2.160 & 2.603 & 2.937 & 3.430 & 3.790 \\
$\sigma_{\pi}^{2}=0.10$ & 0.362 & 0.522 & 0.765 & 1.141 & 1.431 & 2.115 & 2.580 & 2.958 & 3.527 & 3.939 \\
$\sigma_{\pi}^{2}=0.25$ & 0.242 & 0.365 & 0.558 & 0.869 & 1.140 & 1.807 & 2.376 & 2.840 & 3.573 & 4.187 \\
& & & Standard European Call Options & & & \\
$\sigma_{\pi}^{2}=0.00$ & 0.411 & 0.589 & 0.846 & 1.224 & 1.524 & 2.231 & 2.798 & 3.292 & 4.147 & 4.889 \\
$\sigma_{\pi}^{2}=0.10$ & 0.363 & 0.529 & 0.776 & 1.147 & 1.446 & 2.160 & 2.735 & 3.236 & 4.103 & 4.853 \\
$\sigma_{\pi}^{2}=0.25$ & 0.243 & 0.367 & 0.565 & 0.883 & 1.155 & 1.842 & 2.422 & 2.939 & 3.845 & 4.635 \\
\hline \hline
\end{tabular}

Table 2: Knock-out Probabilities of Down-and-Out Call Options (Monthly Frenquency). $S=20, H=16, \lambda=0.03, \mu_{\pi}=0, \sigma_{S}=0.1, \sigma^{2}=\sigma_{S}^{2}-\lambda \sigma_{\pi}^{2}$.

\begin{tabular}{ccccccccccc}
\hline \hline Maturity & $1 / 4$ & $1 / 2$ & 1 & 2 & 3 & 6 & 9 & 12 & 18 & 24 \\
\hline$\sigma_{\pi}^{2}=0.00$ & 0.000 & 0.002 & 0.026 & 0.115 & 0.198 & 0.362 & 0.457 & 0.519 & 0.599 & 0.649 \\
$\sigma_{\pi}^{2}=0.25$ & 0.002 & 0.004 & 0.014 & 0.066 & 0.133 & 0.289 & 0.388 & 0.455 & 0.547 & 0.603 \\
$\sigma_{\pi}^{2}=0.50$ & 0.003 & 0.005 & 0.011 & 0.023 & 0.041 & 0.120 & 0.203 & 0.273 & 0.378 & 0.451 \\
\hline \hline
\end{tabular}


Table 3: Prices of In-the-Money Call Options (Monthly Frenquency). $S=20$, $X=17.5, \lambda=0.03, \mu_{\pi}=0, \sigma_{S}=0.1, \sigma^{2}=\sigma_{S}^{2}-\lambda \sigma_{\pi}^{2}$.

\begin{tabular}{cccccccccccc}
\hline \hline Maturity & $1 / 4$ & $1 / 2$ & 1 & 2 & 3 & 6 & 9 & 12 & 18 & 24 \\
\hline \multicolumn{8}{c}{ Down-and-Out Call Options $(H=16)$} \\
$\sigma_{\pi}^{2}=0.00$ & 2.523 & 2.557 & 2.657 & 2.875 & 3.064 & 3.474 & 3.754 & 3.966 & 4.287 & 4.531 \\
$\sigma_{\pi}^{2}=0.10$ & 2.527 & 2.563 & 2.642 & 2.849 & 3.045 & 3.517 & 3.828 & 4.091 & 4.478 & 4.761 \\
$\sigma_{\pi}^{2}=0.25$ & 2.535 & 2.570 & 2.640 & 2.793 & 2.942 & 3.419 & 3.863 & 4.208 & 4.776 & 5.271 \\
& & & & Standard European Call Options & & & \\
$\sigma_{\pi}^{2}=0.00$ & 2.529 & 2.557 & 2.658 & 2.887 & 3.109 & 3.695 & 4.197 & 4.644 & 5.429 & 6.115 \\
$\sigma_{\pi}^{2}=0.10$ & 2.529 & 2.564 & 2.652 & 2.861 & 3.071 & 3.647 & 4.149 & 4.598 & 5.389 & 6.082 \\
$\sigma_{\pi}^{2}=0.25$ & 2.536 & 2.572 & 2.644 & 2.799 & 2.962 & 3.459 & 3.932 & 4.373 & 5.175 & 5.890 \\
\hline \hline
\end{tabular}

Table 4: Prices of Out-of-the-Money Call Options (Monthly Frenquency). $S=20$, $X=23, \lambda=0.03, \mu_{\pi}=0, \sigma_{S}=0.1, \sigma^{2}=\sigma_{S}^{2}-\lambda \sigma_{\pi}^{2}$.

\begin{tabular}{cccccccccccc}
\hline \hline Maturity & $1 / 4$ & $1 / 2$ & 1 & 2 & 3 & 6 & 9 & 12 & 18 & 24 \\
\hline \multicolumn{8}{c}{ Down-and-Out Call Options $(H=16)$} \\
$\sigma_{\pi}^{2}=0.00$ & 0.001 & 0.015 & 0.088 & 0.293 & 0.507 & 1.088 & 1.561 & 1.949 & 2.549 & 3.003 \\
$\sigma_{\pi}^{2}=0.10$ & 0.014 & 0.032 & 0.095 & 0.280 & 0.478 & 1.046 & 1.515 & 1.925 & 2.585 & 3.086 \\
$\sigma_{\pi}^{2}=0.25$ & 0.032 & 0.064 & 0.124 & 0.269 & 0.418 & 0.910 & 1.398 & 1.820 & 2.551 & 3.212 \\
& & & Standard European Call Options & & & \\
$\sigma_{\pi}^{2}=0.00$ & 0.001 & 0.015 & 0.088 & 0.293 & 0.508 & 1.105 & 1.632 & 2.109 & 2.959 & 3.712 \\
$\sigma_{\pi}^{2}=0.10$ & 0.014 & 0.033 & 0.096 & 0.282 & 0.483 & 1.066 & 1.591 & 2.070 & 2.925 & 3.684 \\
$\sigma_{\pi}^{2}=0.25$ & 0.033 & 0.065 & 0.131 & 0.275 & 0.433 & 0.933 & 1.424 & 1.891 & 2.753 & 3.532 \\
\hline \hline
\end{tabular}


Table 5: Prices of Lookback Call Options (Monthly Frenquency). $S=20, S M=$ $18, \lambda=0.1, \mu_{\pi}=0, \sigma_{S}^{2}=0.25^{2}, \sigma^{2}=\sigma_{S}^{2}-\lambda \sigma_{\pi}^{2}$.

\begin{tabular}{lcccccccc}
\hline \hline Maturity (yrs) & $1 / 4$ & $1 / 2$ & 1 & 2 & 3 & 6 & 9 & 12 \\
\hline$\sigma_{\pi}^{2}=0.00$ & 2.034 & 2.119 & 2.347 & 2.802 & 3.206 & 4.192 & 4.977 & 5.642 \\
$\sigma_{\pi}^{2}=0.10$ & 2.020 & 2.084 & 2.243 & 2.682 & 3.024 & 3.956 & 4.625 & 5.291 \\
$\sigma_{\pi}^{2}=0.25$ & 2.006 & 2.061 & 2.154 & 2.410 & 2.651 & 3.358 & 4.103 & 4.738 \\
\hline \hline
\end{tabular}

